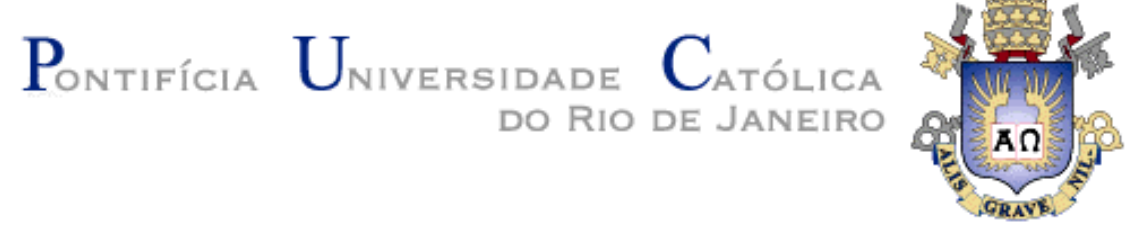

Jorge Marcial Agüero Andrade

\title{
Avaliação Metrológica de Filtros Solares por Espectrometria de Massa LDI-TOF
}

Dissertação de Mestrado

Dissertação apresentada como requisito parcial para obtenção do título de Mestre pelo Programa de PósGraduação em Metrologia para Qualidade e Inovação da PUC - Rio.

Professora Orientadora:

Cássia Ribeiro Ponciano

PósMQI/PUC-Rio

Rio de Janeiro, 19 Setembro de 2008 
Jorge Marcial Agüero Andrade

\section{Avaliação Metrológica de Filtros Solares por Espectrometria de Massa LDI-TOF}

Dissertação apresentada como requisito parcial para obtenção do grau de Mestre pelo Programa de Pós-Graduação em Metrologia Qualidade e Inovação do Centro Técnico Científico da Pontifícia Universidade católica do Rio de Janeiro. Aprovada pela Comissão Examinadora e homologada pela Coordenação Setorial de Pós-Graduação, formalizado pelas respectivas assinaturas:

Comissão Examinadora:

Profa. Dra. Cássia Ribeiro Ponciano Orientadora Programa de Pós-Graduação em Metrologia - PósMQI/PUC-Rio

Profa. Dra. Sônia Renaux Wanderley Louro Departamento de Física - PUC-Rio

Prof. Dr. Ricardo Queiroz Aucélio Departamento de Química-PUC-Rio

Prof. Dr. Enio Frota da Silveira Departamento de Física - PUC-Rio

Coordenação Setorial de Pós-Graduação:

Prof. José Eugenio Leal Coordenador Setorial de Pós-Graduação do Centro Técnico Científico (PUC - Rio)

Rio de Janeiro, 19 de setembro de 2008. 
Todos os direitos reservados. É proibida a reprodução total ou parcial do trabalho sem autorização da universidade, do autor e do orientador.

\section{Jorge Marcial Agüero Andrade}

Graduou-se em Física pela Universidade Nacional Maior de São Marcos do Peru em 1999. Desempenhou atividade profissional e de ensino de 2000 a 2006, atuando na parte de instrumentação física e pesquisa em novos materiais.

Ficha Catalográfica

Agüero Andrade, Jorge Marcial
Avaliação metrológica de filtros solares por
espectrometria de massa LDI-TOF / Jorge Marcial Agüero
Andrade ; orientadora: Cássia Ribeiro Ponciano. - 2008.
93 f. : il. (color.) ; $30 \mathrm{~cm}$
Dissertação (Mestrado em Metrologia para a
Qualidade e Inovação)-Pontifícia Universidade Católica
do Rio de Janeiro, Rio de Janeiro, 2008.
Inclui bibliografia
1. Metrologia - Teses. 2. Filtros solares. 3. LDI-TOF-
4. PDMS. 5. Fator de proteção solar (FPS). 6.
Metrologia química. 7. Regulamentações sobre filtros
solares. I. Ponciano, Cássia Ribeiro. II. Pontifícia
Universidade Católica do Rio de Janeiro. Programa de
Pós-Graduação em Metrologia para a Qualidade e
Inovação. III. Título.

CDD: 389.1 
À minha avó Felicitast, À minha querida mãe Patrícia por todo o apoio e estimulo que rece6o para seguir adiante 


\section{Agradecimentos}

À minha orientadora, Professora Cássia Ribeiro Ponciano, pela confiança e dedicação que contribuíram não só para o desenvolvimento deste trabalho, como também para meu aperfeiçoamento profissional.

Ao coordenador do Programa de Pós-Graduação em Metrologia, Qualidade e Inovação (Pós-MQI), Professor Maurício Nogueira Frota por todo incentivo e sugestões para a realização do Mestrado no Pós-MQI.

À Professora Elisabete Pereira dos Santos da Faculdade de Farmácia da UFRJ, pela doação de amostras de filtros solares e de algumas informações bibliográficas.

À doutora cubana Aurora Pérez Gramatges, pela disposição e ajuda oferecida.

Ao aluno de Iniciação Científica Victor Viana Chaves pela ajuda proporcionada.

À nossa secretária do Pós-MQI, Márcia Ribeiro, pela amizade e sua disposição no trabalho diário.

À PUC-Rio e ao PósMQI pelos auxílios concedidos, sem os quais este trabalho não teria sido realizado.

À Capes pela bolsa concedida para o desenvolvimento deste trabalho.

À minha mãe Patrícia e meus irmãos, pelo constante apoio, e conselhos que sempre recebi para crescer e me superar pessoalmente e profissionalmente.

A todos os meus amigos e colegas do Pós-MQI, pela amizade e companheirismo. 


\section{Resumo}

Agüero Andrade Jorge Marcial. Cássia Ribeiro Ponciano. Avaliação Metrológica de Filtros Solares por Espectrometria de Massa LDI-TOF. Rio de Janeiro, 2008. 93p. Dissertação de Mestrado - Programa de PósGraduação em Metrologia, Qualidade e Inovação (PósMQI), Pontifícia Universidade Católica do Rio de Janeiro.

Diversos trabalhos de pesquisa constatam as evidentes correlações entre a exposição excessiva à radiação solar e a incidência de câncer de pele, de catarata $\mathrm{e}$ de envelhecimento precoce da pele. O mercado de cosméticos destinados à proteção solar encontra-se em franca expansão; diversos países adotam legislações específicas para esses produtos. No Brasil eles são regulados pela Agência Nacional de Vigilância Sanitária (ANVISA). Porém, não existem métodos oficiais de análise química para determinar filtros solares em cosméticos.

No presente trabalho foi desenvolvido um procedimento de análise qualitativa de cosméticos comerciais com filtros solares por espectrometria de massa LDI-TOF (Laser Desorption Ionization - Time-of-Flight). A técnica LDI utiliza um feixe de luz laser monocromático, $\lambda=337 \mathrm{~nm}$, como sonda para excitar, dessorver e ionizar o analito. Embora o espectro da radiação solar seja contínuo, o comprimento de onda utilizado em LDI se situa bem próximo do valor médio da faixa de radiação que compreende UVA e UVB, o que torna a técnica LDI potencialmente apropriada para excitar moléculas das substâncias ativas similarmente aos raios solares. Por tais características, é razoável esperar que LDI seja seletiva para detectar filtros solar presentes nas composições dos cosméticos. Paralelamente, foi utilizada também a espectrometria de massa ${ }^{252} \mathrm{Cf}-\mathrm{PDMS}$ (Plasma Desorption Mass Spectrometry), que utiliza os fragmentos de fissão do nuclídeo radioativo califórnio 252 no lugar do laser. Foram obtidos espectros de massa de íons positivos e negativos de 8 cosméticos comerciais por ambas as técnicas, bem como espectros PDMS das substâncias ativas permitidas pela ANVISA. As massas observadas nos espectros de massa LDI dos produtos selecionados foram comparadas com: i) as massas moleculares de todas as substâncias ativas permitidas; ii) as massas observadas nos espectros PDMS das substâncias "padrões" permitidas e dos cosméticos comerciais; iii) as massas moleculares dos filtros solares indicados nos rótulos.

A seletividade da técnica LDI para identificar filtros solares em cosméticos foi demonstrada pelos espectros de massa de íons positivos e negativos das oito amostras analisadas.

\section{Palavras-chave}

Filtros solares, LDI-TOF-MS, PDMS, Fator de proteção solar (FPS), Metrologia Química, Regulamentações sobre filtros solares 


\section{Abstract}

Agüero Andrade, Jorge Marcial. Cássia Ribeiro Ponciano. Metrological Evaluation of Sunscreens by LDI-TOF Mass Spectrometry. Rio de Janeiro, 2008. 93p. MSc. Dissertation - Programa de Pós-Graduação em Metrologia, Qualidade e Inovação (PósMQI), Pontifícia Universidade Católica do Rio de Janeiro.

Several studies of research note the obvious correlation between excessive exposure to sunlight, and the incidence of skin cancer, cataracts and premature aging of the skin. The market for cosmetics for sun protection is in the booming; various countries adopt laws specific to these products. In Brazil they are regulated by the National Sanitary Surveillance Agency (ANVISA). However, there are no official methods of chemical analysis to determine solar filters in cosmetics.

In this work was developed a procedure for qualitative analysis of commercial cosmetics with solar filters by mass spectrometry LDI-TOF (Laser Desorption Ionization - Time-of-Flight). LDI technique uses a beam of monochromatic laser light, $\lambda=337 \mathrm{~nm}$, as a probe to excite, desorbs and ionize the analyte. Although the spectrum of solar radiation is continuous, the wavelength used in LDI is well on the average range of radiation that includes UVA and UVB, which makes technical LDI potentially suitable to excite molecules of active substances similarly to lightning Sun. For such characteristics, it is reasonable to expect that LDI be selective to detect solar filters presents on cosmetics products. In parallel, was also used mass spectrometry ${ }^{252}$ Cf-PDMS (Plasma Desorption Mass Spectrometry), which uses fragments of the fission of radioactive nuclide californium 252 instead of laser. Mass spectra were obtained from positive and negative ions, eight commercial cosmetics by both techniques, and PDMS spectra of active substances allowed by ANVISA. Masses observed on LDI mass spectra from selected products were compared with: i) molecular masses of all active substances allowed; ii) masses observed on PDMS mass spectra from "standards" allowed and commercial cosmetics; iii) molecular masses of sunscreens in its labels.

The selectivity of the LDI technique to identify solar filters in cosmetics was demonstrated by mass spectra of positive and negative ions of the eight samples.

\section{Keywords}

Sunscreens, LDI-TOF-MS, Solar Protection Factor (SPF), PDMS, Chemical Metrology, Sunscreens regulations. 


\section{Sumário}

1 Introdução

2 Radiação Ultravioleta $\quad 17$

$\begin{array}{ll}2.1 \text { Introdução } & 17\end{array}$

2.2 Faixa Ultravioleta da Radiação Solar 18

$\begin{array}{lll}2.3 & \text { Filtros Solares } & 19\end{array}$

2.3.1 Mecanismo de Ação dos Filtros Solares Químicos 21

2.4 Legislação Vigente 22

2.5 Técnicas Analíticas 24

3 Espectrometria de Massa 25

$\begin{array}{ll}3.1 \text { Princípios } & 25\end{array}$

3.2 Espectrometria de Massa LDI 26

$\begin{array}{ll}3.3 \text { Analisador por Tempo de Vôo } & 28\end{array}$

4 Experimental $\quad 30$

4.1 Espectrômetro de Massa Biflex III Bruker 31

4.1.1 Sistema Laser 31

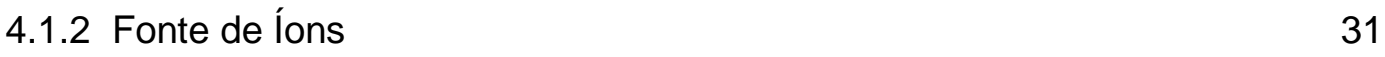

4.1.3 Sistema de Introdução de amostras 32

4.1.4 Refletor 33

4.2 Espectrômetro de Massa ${ }^{252}$ Cf-PDMS 33

4.3 Cosmeticos Analisados por LDI 35

4.4 Cosmeticos Analisados por ${ }^{252} \mathrm{Cf}-\mathrm{PDMS} \quad 35$

4.5 Filtros solares padrões analisados por ${ }^{252} \mathrm{Cf}-\mathrm{PDMS} \quad 35$

5 Resultados 38

5.1 Cosméticos analisados por LDI 38

5.2 Cosméticos analisados por ${ }^{252} \mathrm{Cf}$-PDMS $\quad 47$

5.3 Padrões de filtros solares analisados por ${ }^{252}$ Cf-PDMS 54

6 Discussão e Conclusões $\quad 61$

6.1 Discussão 61 
$\begin{array}{ll}6.2 \text { Conclusões } & 72\end{array}$

$\begin{array}{ll}6.3 \text { Perspectivas } & 74\end{array}$

7 Referências Bibliográficas $\quad 75$

$\begin{array}{ll}\text { Apêndice } & 77\end{array}$

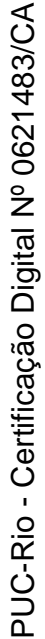




\section{Lista de figuras}

Figura 2.1 - Nomenclatura do espectro eletromagnético, em destaque os comprimentos de onda das três sub-regiões da radiação ultravioleta.

Figura 2.2 - Ester N, N-dimetil PABA: deslocalização do elétron quando absorve radiação UV.

Figura 2.3 - Alguns filtros UV autorizados pela ANVISA classificados em grupos de acordo a sua estrutura química.

Figura 3.1 - Componentes básicos de um espectrometro de massa.

Figura 3.2 - Mecanismo de ação do raio laser na técnica MALDI.

Figura 3.3 - Diagrama esquematico de um espectrômetro de tempo de vôo.

Figura 4.1 - Espectrômetro de massa Biflex III Bruker.

Figura 4.2 - Diagrama do espectrômetro de massa Biflex III Bruker.

Figura 4.3 - Diagrama da fonte de íons SCOUT. O porta amostra é poços é movimentado em um plano vertical de modo que a amostra a ser anlisada fique posicionada sempre no eixo de analisador.

Figura 4.4 - Diagrama do Espectrômetro de massa ${ }^{252} \mathrm{Cf}-\mathrm{PDMS}$.

Figura 4.5 - Espectrômetro de massa ${ }^{252} \mathrm{Cf}-\mathrm{PDMS}$ desenvolvido no laboratório Van de Graff.

Figura 4.6 - Fórmulas estruturais dos filtros padrões analisados por CfPDMS

Figura 5.1 - Espectros de massa LDI de íons positivos e negativos da amostra T1.

Figura 5.2 - Espectros de massa LDI de íons positivos e negativos da amostra T2.

Figura 5.3 - Espectros de massa LDI de íons positivos e negativos da amostra T3.

Figura 5.4 - Espectros de massa LDI de íons positivos e negativos da amostra T4.

Figura 5.5 - Espectros de massa LDI de íons positivos e negativos da amostra E1.

Figura 5.6 - Espectros de massa LDI de íons positivos e negativos da amostra E2. 
Figura 5.7 - Espectros de massa LDI de íons positivos e negativos da amostra E5.

Figura 5.8 - Espectros de massa LDI de íons positivos e negativos da amostra Lip care.

Figura 5.9 - Espectros de massa ${ }^{252}$ Cf-PDMS de íons positivos e negativos da amostra T1.

Figura 5.10 - Espectros de massa ${ }^{252} \mathrm{Cf}-\mathrm{PDMS}$ de íons positivos e negativos da amostra T2.

Figura 5.11 - Espectros de massa ${ }^{252} \mathrm{Cf}-\mathrm{PDMS}$ de íons positivos e negativos da amostra T3.

Figura 5.12 - Espectros de massa ${ }^{252} \mathrm{Cf}-\mathrm{PDMS}$ de íons positivos e negativos da amostra T4.

48

Figura 5.13 - Espectros de massa ${ }^{252} \mathrm{Cf}-\mathrm{PDMS}$ de íons positivos e negativos da amostra E1.

Figura 5.14 - Espectros de massa ${ }^{252} \mathrm{Cf}$-PDMS de íons positivos e negativos da amostra E5.

Figura 5.15 - Espectros de massa Cf-PDMS de íons positivos e negativos da amostra padrão Eusolex 9020.

Figura 5.16 - Espectros de massa Cf-PDMS de íons positivos e negativos da amostra padrão octocrylene.

Figura 5.17 - Espectros de massa Cf-PDMS de íons positivos da amostra padrão Eusolex 232.

Figura 5.18 - Espectros de massa Cf-PDMS de íons positivos da amostra padrão octilmetoxinamato.

Figura 5.19 - Espectros de massa Cf-PDMS de íons positivos da amostra padrão benzofenona 3 .

Figura 5.20 - Espectros de massa Cf-PDMS de íons positivos e negativos da amostra padrão Uvinul MS40.

Figura 5.21 - Espectros de massa Cf-PDMS de íons positivos da amostra padrão Eusolex 6007. 


\section{Lista de tabelas}

$\begin{array}{lll}\text { Tabela 2.1 Faixas da radiação ultravioleta segundo a CIE. } & 18\end{array}$

Tabela 2.2 Exemplo de filtros solares químicos e físicos. 20

Tabela 2.3 Faixas de absorção e comprimento de onda crítico para os filtros UV usados habitualmente. 20

Tabela 4.1 Filtros solares padões analisados 36

Tabela 5.1 Massas de íons positivos correspondentes aos principais picos dos espectros de massa LDI de amostras dos cosméticos analisados.

Tabela 5.2 Massas de íons negativos correspondentes aos principais picos dos espectros de massa LDI de amostras dos cosméticos analisados.

Tabela 5.3 Massa de íons positivos correspondentes aos principais picos dos espectros de massa Cf-PDMS dos cosméticos analisados.

Tabela 5.4 Massas de íons negativos correspondentes aos principais picos dos espectros de massa Cf-PDMS.

Tabela 5.5 Massas de íons positivos correspondentes aos principais picos dos espectros de massa Cf-PDMS dos padrões analisados.

Tabela 5.6 Massas de íons negativos correspondentes aos principais picos dos espectros de massa Cf-PDMS dos padrões analisados.

Tabela 6.1 Relação de filtros solares permitidos pela ANVISA em ordem crescente de massas moleculares. Os compostos em negrito foram analisados neste trabalho por Cf-PDMS. 


\section{Lista de abreviaturas e Símbolos}

ANVISA - Agência Nacional de Vigilância Sanitária

ABIHPEC - Associação Brasileira da Indústria de Higiene Pessoal

CAS - Chemical Abstract Service

COLIPA - European Cosmetic, Toiletry and Perfumery Association, Representa os interesses coletivos das indústrias deste setor estabelecidas na União Européia. Corresponde à ABIHPEC no Brasil

DESORPTION - Dessorção: Transferência de átomos, moléculas ou agregados de um sólido para a fase gasosa

FDA - Food and Drug Administration

FPS - Fator de proteção solar

IFRA Índex - International Fragance Cosmetic

$\mathrm{INCl}$ - International Nomenclature Cosmetic Ingredient

INMETRO - Instituto Nacional de Metrologia, Normalização e Qualidade Industrial

LDI - Laser desorption ionization

MALDI - Matrix Assisted laser desorption ionization

OTC - (Over the Counter) Medicamento de venda livre

Sci-Finder - Programa de busca de informação cientifica.

RIV - Radiação Infravermelha

RUV - Radiação Ultravioleta 